Table 1. Pulmonary function test results

Pulmonary function tests

Pre-treatment Post-treatment

Forced expiratory volume

77.65

82.12

1 / forced vital capacity

Forced expiratory volume 1, L 1.95 (46\%)

$2.98(70.6 \%)$

Forced vital capacity, L

$2.51(50 \%)$

$3.98(80 \%)$

Total lung capacity, L

$4.19(62 \%)$

$5.2(77 \%)$

Residual volume, $\mathrm{L}$

$1.33(84 \%)$

$1.40(88 \%)$

Carbon monoxide transfer

$1.18(67 \%)$

$1.50(87 \%)$

coefficient (KCO)

Transfer capacity of the lung $5.25(45 \%) \quad 8.91(77 \%)$

for the uptake of carbon

monoxide (TLCO)

significantly raised at more than $200 \mathrm{IU} / \mathrm{mL}$ (normal range $<20$ ) even 6 months after stopping infliximab.

\section{Conclusion}

CEP is extremely rare and should be considered in patients receiving infliximab who develop eosinophilia, and pulmonary infiltrates with dyspnoea. Infliximab antibodies should always be checked before commencing infliximab therapy. To our knowledge there are only three reported cases of pulmonary eosinophilia with infliximab therapy, but all those patients developed eosinophilia acutely. ${ }^{5,6}$ Our patient developed symptoms 3 months after discontinuation of infliximab therapy. This makes it the first reported case of delayed reaction to infliximab leading to CEP.

\section{References}

1 Corominas M, Gastaminza G, Lobera T. Hypersensitivity reactions to biological drugs. J Investig Allergol Clin Immunol 2014;24:212-25.

2 Keane J, Gershon S, Wise RP et al. Tuberculosis associated with infliximab, a tumor necrosis factor alpha-neutralizing agent. N Engl J Med 2001;345:1098-104.

3 Warris A, Bjorneklett A, Gaustad P. Invasive pulmonary aspergillosis associated with infliximab therapy. N Engl J Med 2001;344:1099-100.

4 Panagi S, Palka W, Korelitz B et al. Diffuse alveolar hemorrhage after infliximab treatment of Crohn's disease. Inflamm Bowel Dis 2004;10:274-7.

5 Rubin DT, Sohi S, Shilling RA, White SR. Pulmonary eosinophilia following infliximab treatment for Crohn's disease. Gastroenterol Hepatol 2006;2:592-6.

6 Baig I, Storch I, Katz S. Infliximab induced eosinophilic pleural effusion in inflammatory bowel disease. Am J Gastroenterol 2002; 97:S177.

Address for correspondence: Dr Iftikhar Nadeem, Bedford Hospital, Bedford Hospitals NHS Foundation Trust, Kempston Rd, Bedford MK40 9DJ, UK.

Email: Iftikharnadeem49@gmail.com

\title{
Lessons of the month 3: ST-elevation myocardial infarction and left ventricular thrombus formation: an arterial thrombotic complication of severe COVID-19 infection
}

Authors: Matthew Fenton, ${ }^{A}$ Seshnag Siddavaram, ${ }^{B}$ Conn Sugihara ${ }^{C}$ and Syed Husain ${ }^{D}$

We describe a case of an 82-year-old man who developed an anterior ST-elevation myocardial infarction (STEMI) and left ventricular thrombus while an inpatient following a diagnosis of severe COVID-19 infection (SARS-CoV-2). His D-dimer was significantly elevated at $12,525 \mathrm{ng} / \mathrm{mL}$ (normal range $<243$ ). He unfortunately died despite management with thrombolysis,

Authors: ${ }^{A}$ core medical trainee year 2, Maidstone Hospital, Maidstone, UK; ${ }^{B}$ acute medical specialist registrar year 4 , Maidstone Hospital, Maidstone, UK; ' Cardiology consultant, Maidstone Hospital, Maidstone, UK; Drespiratory consultant, Maidstone Hospital, Maidstone, UK warfarin and non-invasive ventilation. This case provides an example of a likely arterial thrombotic complication of severe COVID-19 infection. Clinicians should be aware of this possibility in such patients, with a severely prothrombotic state as a possible underlying aetiology. Further research is required to establish any causative link, pathophysiological mechanisms and whether modification to existing venous thromboembolism prophylaxis strategies may also reduce arterial thrombotic complications of severe COVID-19 infection.

KEYWORDS: COVID-19, myocardial infarction, ventricular thrombus, arterial thrombosis, D-dimer

DOI: $10.7861 /$ clinmed.2020-0266 


\section{Case presentation}

An 82-year-old man was admitted following a 10-day history of a productive cough of green sputum, accompanied by fevers. He had also become increasingly short of breath over the 2 days prior to admission. He did not experience any chest pains prior to admission.

He had a background of a total knee replacement. He was not hypertensive and had recent normal glycated haemoglobin (HbA1c) and total cholesterol levels, with no history of ischaemic heart disease. He had a 10 pack-year smoking history and drank 10 units/week of alcohol. There was no significant family history. Prior to admission he lived at home independently, his Rockwood Clinical Frailty Scale score was 2 and he had a good exercise tolerance of approximately 1 mile.

On arrival he was hypoxic (oxygen saturations were $71 \%$ on room air and $96 \%$ on $40 \%$ oxygen via Venturi mask) and tachypnoeic (26 breaths/minute). His heart rate was 71 beats/minute, blood pressure $132 / 81 \mathrm{mmHg}$ and temperature $36.5^{\circ} \mathrm{C}$. Examination revealed increased work of breathing and bilateral fine inspiratory crepitations to the mid-zones on auscultation.

His arterial blood gas showed type 1 respiratory failure on $60 \%$ fraction of inspired oxygen $\left(\mathrm{FiO}_{2}\right)$. Blood tests revealed a significantly raised C-reactive protein (CRP) of $412 \mathrm{mg} / \mathrm{L}$ (normal range $0-5$ ). His chest $X$-ray demonstrated severe diffuse bilateral infiltrates (Fig 1) and his admission electrocardiography was unremarkable. COVID-19 infection with SARS-CoV-2 was highly suspected and a naso-pharyngeal polymerase chain reaction swab confirmed this diagnosis.

After 36 hours of admission, he developed sudden onset central crushing chest pain on the medical ward. Electrocardiography revealed dynamic marked ST-segment elevation in leads V1-4, with reciprocal changes in the lateral leads, consistent with an acute anterior ST-elevation myocardial infarction (STEMI; Fig 2). Troponin T was raised at $354 \mathrm{ng} / \mathrm{L}$ (range 0-14) and 9 hours later increased to 4,549 ng/L. Subsequent electrocardiography revealed deep Q waves in leads V1-4 and the inferior leads. His case was discussed with the regional primary percutaneous coronary intervention (PPCI) centre. Given his pre-existing oxygen requirement, it was felt that he would require invasive ventilation to facilitate transfer to a different hospital site for PPCI. After

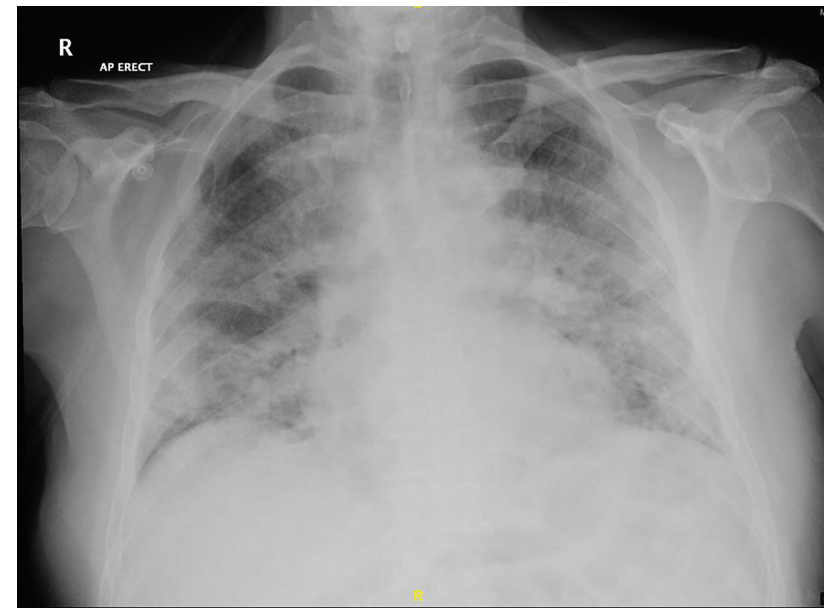

Fig 1. Chest X-ray demonstrating severe diffuse bilateral infiltrates.

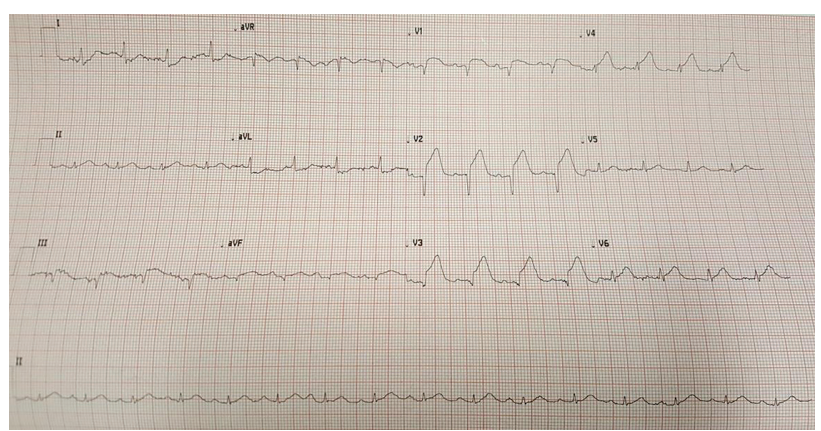

Fig 2. Electrocardiography revealing dynamic marked ST-segment elevation in leads V1-4, with reciprocal changes in the lateral leads, consistent with an acute anterior ST-elevation myocardial infarction.

discussion with local and regional cardiologists, intensivists and physicians, it was decided that he was not a candidate for intubation or resuscitation. A decision was made to treat locally with thrombolysis with 8,000 units of intravenous tenecteplase, $300 \mathrm{mg}$ of aspirin, $300 \mathrm{mg}$ of clopidogrel, intravenous morphine and an intravenous glyceryl trinitrate infusion. He demonstrated a partial response with a marked reduction in his chest pains and remained haemodynamically stable.

He was initiated on non-invasive ventilation using continuous positive airway pressure (CPAP) with $75 \% \mathrm{FiO}_{2}$ and positive endexpiratory pressure of $10 \mathrm{cmH}_{2} \mathrm{O}$. He was also started on $1.25 \mathrm{mg}$ of bisoprolol and $1.25 \mathrm{mg}$ of ramipril once daily, subcutaneous $60 \mathrm{mg}$ of enoxaparin twice daily and $80 \mathrm{mg}$ of atorvastatin once daily.

Further tests revealed a significantly elevated D-dimer at 12,525 $\mathrm{ng} / \mathrm{mL}$ (normal range $<243$ ) and raised lactate dehydrogenase at 1,635 IU/L (normal range 240-480). He was lymphopenic for the majority of the admission (nadir $0.66 \times 10^{9} / \mathrm{L}$ ). His peak CRP was $501 \mathrm{mg} / \mathrm{L}, 9$ hours after chest pain onset.

A transthoracic echocardiography was performed and revealed a moderately impaired left ventricular ejection fraction (approximately $40 \%$ ), a left ventricular aneurysm and regional wall motion abnormalities (basal septal and apical wall hypokinesis). A small left ventricular thrombus was also detected measuring $0.8 \times 1.1 \mathrm{~cm}$ (Fig 3). The right ventricle appeared normal with

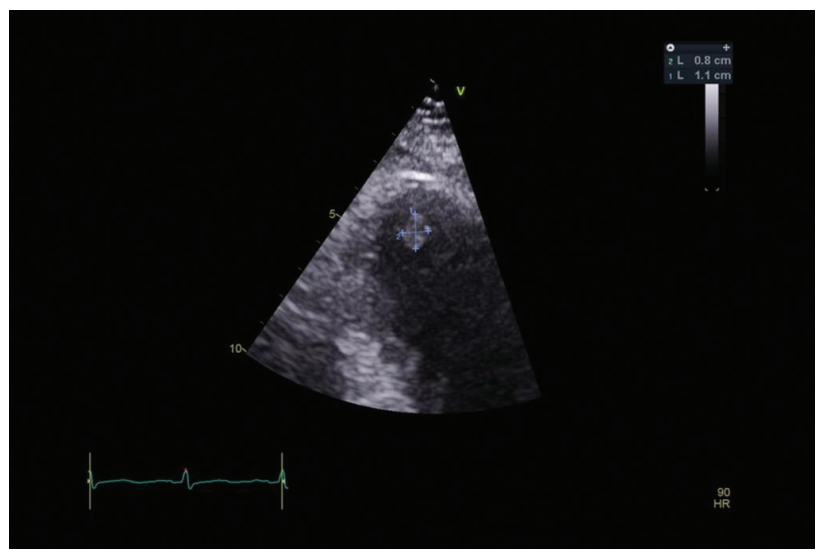

Fig 3. A small left ventricular thrombus was also detected measuring $0.8 \times 1.1 \mathrm{~cm}$. 
no significant valvular abnormalities. Due to the left ventricular thrombus, warfarin was initiated with a target international normalised ratio of 2-3.

As he was not a candidate for invasive ventilation, he received a prolonged CPAP course of 14 days. Unfortunately, this could not be weaned, with a persistent $\mathrm{FiO}_{2}$ requirement of $>55 \%$. His dependence on CPAP meant coronary angiography could not be performed. A repeat chest $\mathrm{X}$-ray demonstrated worsening diffuse bilateral infiltrates. $\mathrm{His} \mathrm{FiO}_{2}$ increased to $95 \%$ despite a trial of antibiotics and diuresis, so a decision was made for palliative care and withdrawal of CPAP treatment. He sadly died on the 16th day of his admission.

\section{Discussion}

We describe a case of a left ventricular thrombus associated with an STEMI in a patient with severe COVID-19 infection, who subsequently died. We suspect that these arterial thrombotic events were complications of severe COVID-19 infection. A recent case series described 18 patients with ST-segment elevation and COVID-19 infection.' Like our patient, all these patients had a significantly raised D-dimer, which is an unusual finding in acute myocardial infarction. Notably, eight out of 18 patients in this series suffered a STEMI after developing confirmed COVID-19 infection. Another recent case series described five large vessel ischaemic strokes in patients with an age range of 33-49, who were all also diagnosed with COVID-19 on admission. ${ }^{2}$ Three of these patients also had a significantly elevated D-dimer.

A significant prothrombotic state in severe COVID-19 infection has been described, with a combination of severe inflammation, cytokine storm, immobility and disseminated intravascular coagulopathy. ${ }^{3,4}$ In one case series of 184 COVID-19 patients on intensive care units, $27 \%$ had a venous thromboembolic event and $3.7 \%$ had an arterial thrombotic event despite standard prophylaxis. ${ }^{5}$ Furthermore, thrombotic microvascular injury and endothelial cell damage have been evident on post-mortem studies of severe COVID-19 patients. ${ }^{6}$

Managing patients with confirmed severe COVID-19 and acute myocardial infarction provides a significant management challenge. The finding of a left ventricular thrombus in this case is consistent with a strongly prothrombotic state. It may be more important to screen for left ventricular thrombus in such patients. A significant prothrombotic state in severe COVID-19 infection appears to increase the risk of both venous and arterial thrombotic events, which can be fatal. It is possible that COVID-19 specific thromboprophylaxis regimens may reduce both venous thromboembolism rates and acute cardiovascular events such as myocardial infarction and stroke in the context of severe
COVID-19 infection. Further research is required in this area and also to explore whether modulation of acute proinflammatory pathways or the immune response would reduce acute thrombotic complications of severe COVID-19.

\section{Learning points}

> Severe COVID-19 infection appears to be linked to a prothrombotic state which may cause severe arterial thrombotic complications including STEMI and ventricular thrombus formation. Clinicians should be alert to this possibility when managing such cases. Further studies are required to establish any causative link and any pathophysiological mechanisms.

> Further research is required to determine optimal anticoagulation strategies in severe COVID-19 patients for prevention and treatment of venous/arterial thromboses.

> Patients with STEMI in the context of severe COVID-19 infection appear to often have a significantly elevated D-dimer. This is not normally a feature of acute myocardial infarction and may reflect an underlying prothrombotic state.

\section{Acknowledgements}

We thank to the patient's family for allowing us to publish this case report.

\section{References}

1 Bangalore S, Sharma A, Slotwiner A et al. ST-Segment elevation in patients with COVID-19 - a case series. N Engl J Med 2020:NEJMc2009020 [Epub ahead of print].

2 Oxley TJ, Mocco J, Majidi S et al. Large-vessel stroke as a presenting feature of COVID-19 in the young. N Engl J Med 2020;382:e60.

3 Chen T, Wu D, Chen H et al. Clinical characteristics of 113 deceased patients with coronavirus disease 2019: retrospective study. BMJ 2020;368:m1091.

4 Mehta P, McAuley D, Brown M et al. COVID-19: consider cytokine storm syndromes and immunosuppression. Lancet 2020;395:1033-4.

5 Klok F, Kruip M, van der Meer $\mathrm{N}$ et al. Incidence of thrombotic complications in critically ill ICU patients with COVID-19. Thromb Res 2020;191:145-7.

6 Magro C, Mulvey J, Berlin D et al. Complement associated microvascular injury and thrombosis in the pathogenesis of severe COVID-19 infection: A report of five cases. Transl Res 2020;220:1-13.

Address for correspondence: Dr Syed Husain, Respiratory Department, Travers Unit, Maidstone Hospital, Hermitage Lane, Maidstone, Kent ME16 9QQ, UK.

Email: syedhusain@nhs.net 